\title{
Acceptance of novel food by horses: the influence of food cues and nutrient composition
}

M. van den Berg ${ }^{1 \dagger}$, V. Giagos ${ }^{2}$, C. Lee ${ }^{3}$, W.Y. Brown ${ }^{1}$, and G.N. Hinch ${ }^{1}$

${ }^{1}$ School of Environmental and Rural Science, University of New England, Armidale NSW 2351, Australia

${ }^{2}$ School of Science and Technology, University of New England, Armidale NSW 2351, Australia

${ }^{3}$ CSIRO, Agriculture, Armidale NSW 2350, Australia

${ }^{\dagger}$ Corresponding author: Mariette van den Berg. Email: info@mbequineservices.com 


\section{Abstract}

2 Compared to ruminants little is known about how horses modulate food intake and learn about

3 flavour-to-post-ingestive consequences. While it has been suggested that due to hindgut

4 fermentation horse's foraging preferences may be largely influenced by sensory input (e.g.

5 volatiles), it has been established that horses are able to differentiate and select familiar foods (e.g.

6 concentrates and hay) based on nutritional content. Yet it remains unclear how this translates to the

7 acceptance of nutritious novel foods (NF). Therefore, the influence of food cues and nutrient composition on NF acceptance were examined in two experiments using 11 adult mares. In experiment 1, we investigated the influence of a familiar odour (FO) on the acceptance of a nutritious NF and in experiment 2, we determined if horses have the ability to select nutritious NF based on the nutritional content, regardless of sensory preferences. In experiment 1 horses received identical NF in a two-choice test with one of the choices being masked with a FO over a 9-day period. In experiment 2 horses were offered a high or low protein option of an otherwise identical NF in a two-choice test in which the NFs were paired with two unfamiliar flavours (odours). The two-choice test lasted for 14 days and the flavour-protein pairing was switched after 7 days. NF intakes were recorded over a 10 min test period on each test day and analysed using Bayesian hierarchical models. The results of experiment 1 indicate that a FO had a strong positive influence on the NF intake for the first 5 days (90-100\% of total consumption and strong evidence for nonzero temporal effects (Bayes factor $\mathrm{B}_{12}=110$ )). This was followed by a more even distribution of intake for the remaining period. In experiment 2 horses had a greater intake of high protein NF regardless of the flavour on days 4, 6 and 7 (80-87\% of total consumption) and this continued after the switch over (Day 9 to $14 ; 57-81 \%$ of total consumption). However, 4 out of 11 horses showed neophobia throughout the testing period, which could have been associated with the novel odours as horses scanned the buckets with little to no sampling. The results suggest that pre-ingestive cues 
acceptance of NF. This new knowledge could be applied by the horse industry to encourage the consumption of new food or forages by horses.

\section{Keywords}

Diet Selection, Flavour, Food Neophobia, Horses, Odour, Novel Food

\section{Introduction}

The senses of taste and smell help animals to discriminate between foods and play an important role in food preferences and food selection. Orosensory senses together with post-ingestive mechanisms allow animals to make associations with pleasant or unpleasant experiences depending on whether the effect on the internal environment of the animal is aversive or positive (gut-brain feedback) (Provenza, 1995). For example foods can be rejected (aversion) as a direct result of sensory input and its link to post-ingestive consequences, either from toxins or nutrients that are in excess or deficient. Learned food aversions have been demonstrated in a number of animal species including ruminants (Burritt and Provenza, 1991; Provenza, 1995; 1996), rats (Garcia et al., 1972) and horses (Houpt et al., 1990; Pfister et al., 2002). Conversely, the liking of a food (preference) increases when it contains adequate nutrients and provides a positive feedback.

Investigations of the ability of horses to differentiate foods based on the orosensory characteristics and nutrient content are limited. There is some evidence that horses are able to detect macronutrients in foods and can adapt to deficiencies by increasing intake or by changing food choices (Laut et al., 1985; Cairns et al., 2002; Redgate et al., 2014). For example, Cairns et al. (2002) showed that horses selected a higher energy concentrate over a lower energy one, regardless of the preferred flavour (mint or garlic). However, it has been suggested that horses, due to hindgut fermentation, may experience difficulties in associating the chosen food with its post-ingestive 
consequences, particularly when several foods are presented simultaneously. Therefore, a more recent study compared the effect of exposure to a single forage that was rich in either protein, lipids or hydrolysable carbohydrates on the selection of three forages in a simultaneous choice session (Redgate et al., 2014). The horses showed a greater preference for the forages that were rich in protein or hydrolysable carbohydrates, which suggests that horses responded to the macronutrients in the diets and that the dietary experience (single presentation) facilitated feedback mechanisms, and hence affected dietary preferences.

However, other researchers have suggested that diet selection and intake are more influenced by the organoleptic qualities of forages (e.g. taste, odour, ease of prehension, texture) and that nutrient content appeared to be weak indicators (Dulphy et al., 1997; Cuddeford, 2005). Food selection based on orosensory perception has been observed in a previous study (van den Berg et al., 2016) that examined the effect of energy status (low and high) in horses on diet selection of familiar and novel forages. These findings demonstrate that horses had a greater preference for familiar forages and that, regardless of the energy intake, horses showed a strong neophobic response towards unfamiliar nutritious forages, frequently scanning the buckets with little to no consumption. These novel forages had comparable nutritional profiles to the familiar forages and therefore it seems that volatiles and odour (olfaction) may be important factors in forage selection by horses, in particular when dealing with a familiar-novel dichotomy. The influence of plant odours on herbivore feeding behaviour and dietary preferences has been clearly demonstrated in sheep (Arnold et al., 1980). In addition, odour profiling has been used to make predictions about the preferences (and links with nutritional traits) for familiar forages (oat and lucerne hay) by horses and dairy cattle (Pain and Revell, 2009).

While it has been established that horses are able to make associations with the nutritional content of familiar foods, it is unclear how this applies to the selection of nutritious novel foods and how 
volatiles may affect this selection. Therefore, the aim of the present study was to improve our understanding of the acceptance of nutritious novel foods by horses by examining the sensory behaviour and the ability of horses to learn about positive post-ingestive consequences of a novel food in two experiments. The first experiment follows our previous study (van den Berg et al., 2016) that demonstrated a strong neophobic response in horses towards novel forage volatiles and focused on the question of whether odour influences diet selection of novel foods by horses. It was hypothesised that horses would cautiously sample all foods on offer, but initially a greater intake was expected for the novel choice with the familiar odour. The second experiment focused on the question of whether horses make associations with the orosensory characteristics of an unfamiliar nutritious food and their post-ingestive consequences. The hypothesis was that horses would cautiously sample all foods available, but a greater intake for food with a superior nutrient profile was expected regardless of the preferred flavours.

\section{Material and methods}

\subsection{Animals and feeding management}

A total of 11 healthy horses were used for the study. Horses were managed at a commercial horse facility in the New England region (NSW, Australia). The mares were between the ages of 3 and 15 years, weighing 480-640 kg and were of Australian Stock Horse $(n=8)$ and Thoroughbred $(\mathrm{n}=3)$ breeds. Horses initially were grazing pasture and had a Henneke's body condition score between 4 and 8 (moderately thin to fat, Henneke et al., 1983). The management and feeding of horses was based on the horse owner's usual practices and throughout the study period horses were managed on pasture as one group and were only offered lucerne (Medicago sativa) hay ad libitum to supplement poor winter pasture. The horses were not exercised, apart from one horse that was ridden (light work) on three occasions during the experiment period (after the tests). This horse did 
not receive any additional supplementation. The study was conducted between the months of July and October 2014.

\subsection{Experimental design}

In the first experiment horses received identical novel forages in a two-choice test for 9 days with one of the choices being masked with a familiar odour (Figure 1). The aim was to examine intake patterns and the time required for horses to learn about the post-ingestive consequences of the novel foods. We propose that a positive experience can be assumed when horses consumed 50\% or more of the total food offered and consumed equal portions of both choices.

In the second experiment horses were offered novel foods from a similar source in a two-choice test that were high or low in protein paired with one of two unfamiliar flavours. The two-choice tests were conducted over 14 days with the flavour-protein pairing switched after 7 days (Figure 1). The objective was to examine intake patterns and the time taken for horses to increase consumption of the high-protein food (post-ingestive feedback) and if horses would continue to make this selection when flavour-protein pairing was switched.

\subsection{Feed collection and flavour preparation}

Golden bamboo (Phyllostachys aurea) was chosen as the test forage for experiment 1 based on the novelty and literature describing the use as supplementary fodder in horse diets (Nelson, 1997; Triebe et al., 2012; van den Berg et al., 2016). Bamboo was sourced from the nursery of the University of New England, NSW. The browse was stripped to leaves and twigs, dried in a climatecontrolled room at $27^{\circ} \mathrm{C}$ for 3-4 days and cut in pieces similar to the familiar chaff form $(2-3 \mathrm{~cm})$. 
130 For the preparation of forage odour a similar procedure was used as described by Hinch et al.

131 (2004). Fresh lucerne was collected from a commercial lucerne grower in South East Queensland,

132 Australia. For the familiar odour solution $200 \mathrm{~g}$ fresh lucerne was shredded in a food blender with 1

$133 \mathrm{~L}$ water to make up $20 \mathrm{~L}$ of total solution. This was allowed to stand overnight $(12 \mathrm{~h})$ in a cool

134 room at $4^{\circ} \mathrm{C}$ before straining through a cheese-cloth. The resultant extract was stored at $-20^{\circ} \mathrm{C}$ in

135 airtight containers.

137 Commercially sourced non-nutritive human/animal food flavours (aniseed and citrus; Lucta, Spain)

138 were used in experiment 2. The aniseed flavour had a liquorice aroma and citrus had a sweet orange

139 aroma. Both flavours had no added taste and were novel to the horses. The flavour powder $(5 \mathrm{~g})$

140 was diluted in $500 \mathrm{ml}$ cold water to make a stock solution.

2.4. Testing area

Horses were individually tested in a holding yard $(4 \mathrm{~m} \times 8 \mathrm{~m})$ that was familiar to them and within view of other horses. Before testing (experiment 1) horses were adapted to the holding yard and buckets for two days during which they were fed lucerne chaff (Figure 1). Two large feeding buckets were mounted on the yard door $(0.5 \mathrm{~m}$ apart and $1 \mathrm{~m}$ height $)$ and two smaller labelled feeding tubs were placed inside the larger mounted buckets. The position of the feeding tubs was changed randomly for each testing day.

\subsection{Testing procedures}


Horses were offered two identical feeding buckets, each containing $200 \mathrm{~g}$ bamboo chaff (novel forage) with and without a FO (lucerne). The forages were placed on mesh cloths that acted as the floor of each feeding tub; the mesh size prevented the foods from falling through while allowing any odour below to permeate the food. To deliver the FO, $200 \mathrm{ml}$ of the lucerne extract was placed $3.5 \mathrm{~cm}$ below the feeder base. In addition, the mesh cloth of the bucket with the FO was drenched in the lucerne solution and dried overnight. Each day new cloths were prepared for the feeding tubs. The labelled feeding tubs were rinsed with water and dried with paper tissue between each horse and test. Feeding tubs used with the FO were kept separate from the other feeding tubs.

\subsubsection{Experiment 2: Nutrient composition (Post-ingestive feedback)}

Horses were offered two novel foods ( 2 x $200 \mathrm{~g}$; soybean hull pellets) that contrasted in crude protein (CP) levels $(13.9 \%$ vs. $22.3 \%)$. The two diets were paired with one of two unfamiliar flavours (aniseed or citrus odours). Commercially sourced soybean hull pellets (SHP) with a CP level of $13.9 \%$ and digestible energy content of $8.4 \mathrm{MJ} / \mathrm{kg}$ on a dry matter (DM) basis constituted the low protein (LP) option. Soybean meal $(65 \mathrm{~g} ; 47.5 \% \mathrm{CP})$ was added to the soybean hulls to create a high protein (HP) option $(22.3 \% \mathrm{CP})$ with minimal change to the fibre content and volume. This meant that the total offered was $265 \mathrm{~g}$ for the high CP option, which resulted in a digestible energy of $9.9 \mathrm{MJ} / \mathrm{kg}$ for HP diet. The flavour stock solutions ( $3 \mathrm{ml}$ citrus or $5 \mathrm{ml}$ aniseed) were further diluted in water $(300 \mathrm{ml})$ before adding to the novel foods to dampen the feed and create a homogenous mixture. This concentration created an aromatic odour that was detectable by human senses. Horses were randomly allocated to a flavour-protein pairing based on age and estimated weight/ body condition score as this allowed for distribution of treatments across testing days and eliminated the influences of potential weather conditions for one particular treatment. At the start of the experiment 6 horses received HP-aniseed/LP-citrus and 5 horses HP-citrus/LP-aniseed. The flavour-protein pairing was switched after 7 days. 
The two-choice tests (10 $\mathrm{min})$ were conducted between 09:00 and 12:30 h each day of the testing

185 period. The intake of NF by each horse was determined by weighing the foods in each feeding bucket before and after each test. For the SHP trial (experiment 2) the intake was adjusted for moisture (added water) and calculated to a DM basis. On completion of testing horses were allowed to return to their pasture paddock.

\subsection{Statistical analysis}

All the statistical analyses were performed with the R3.1.3 free software (R Core Team, 2015). Additional information about the statistical analysis used in this study is covered in the supplementary material supplied.

\subsubsection{Experiment 1: Familiar odour}

To determine if there was an effect of familiar odour on the intake of a novel forage and if horses learn about the nutritional consequences of novel forages over time, the intake (g, DM) and proportions (percentage) of bamboo without lucerne odour $\left(\mathrm{BO}^{-}\right)$and bamboo with lucerne odour $\left(\mathrm{BO}^{+}\right)$over the 9 testing days were examined. We denoted the proportion of $\mathrm{BO}^{+}$intake out of the total intake of the $i$-th horse and the $j$-th day with $p_{i j}$ and we chose a logistic link function with our covariates. Our first covariate was an intercept $\beta_{0}$, which expressed a main effect and followed a Normal distribution with mean zero and variance $1000^{2}$. The zero mean indicates that overall the horses had a balanced consumption (distribution of 50\% from each bucket) whereas the large prior variance allowed us to consider a wider range of common behaviours across all horses and days. 
208 The experimental design suggested two consistent sources of variation: a random effect, which could be attributed to each different horse and a temporal effect where each measurement depended on the day of the observation. In previous ruminant studies (Launchbaugh et al., 1997; Van Tien et al., 1999; Hinch et al., 2004) novel food acceptance (reduction in neophobia) was measured as a transition from very limited consumption of a novel food to an incremental increase in intake that could reach total offered amounts (plateau) depending on the post-ingestive feedback. We hypothesised that this would be the same for horses and therefore the inclusion of the temporal effects expresses this transition, which we assume is common for all horses. Whereas, the inclusion of the random effects expresses the horse-specific variation (e.g. age, body condition score).

We addressed both sources of variation using a Bayesian hierarchical model. Each horse's foraging behaviour was modeled with a random variable $\left(\beta_{h}\right)$ which followed a Normal distribution with a mean 0 and variance which in turn followed a Half-Cauchy prior (Gelman, 2006). The heavy-tailed prior on the variance allowed us to explore a wide class of models for the foraging behaviour (consistent vs. variable) of each horse. Similarly, for the temporal effect we have chosen a Normaldistributed random variable $\left(\beta_{d}\right)$ for each day with mean 0 and variance $\sigma_{d}^{2}$ with a Half-Cauchy prior as well. The residual terms $\epsilon_{i j}$ followed a Normal distribution with mean 0 and variance $\sigma_{\epsilon}^{2}$.

Other choices were considered (linear model, lineal change-point model, auto-regressive model) but the aforementioned choice had the smallest deviance information criterion (DIC) (Spiegelhalter et al., 2002) with the linear change-point (Day 6) model having a slightly increased DIC. Overall the hierarchical model was:

$\beta_{h i} \sim \operatorname{Normal}\left(0, \sigma_{h}^{2}\right) \quad \sigma_{h}^{2} \sim$ Half-Cauchy $(0,1) \quad \beta_{d j} \sim \operatorname{Normal}\left(0, \sigma_{d}^{2}\right) \quad \sigma_{d}^{2} \sim$ Half-Cauchy $(0,1)$ 
We implemented this model in JAGS (Plummer, 2003) and processed the output in R using rJAGS 234 (Plummer and Stukalov, 2014).

To establish if horses make associations with the orosensory characteristics of a novel food and their post-ingestive consequences, the intake (g, DM) and proportions (percentage) of SHP based on protein levels and flavour over 14 testing days were examined. Only observations of seven horses were used in this analysis, as 4 of the 11 horses did not have an intake of $25 \mathrm{~g}$ SHP or more after Day 4 and remained neophobic throughout the testing period. Our measurements consisted of intakes (g, DM) of SHP, which contained either HP, or LP and could have either a citrus or aniseed flavour. Both the protein and the flavour have been treated as two-level factors. Our parameter of interest is the proportion of HP-SHP out of the total intake. During the first 7 days 4 horses received HP-aniseed/LP-citrus and 3 horses HP-citrus/LP-aniseed. The flavour-protein pairing was switched after 7 days.

Similar to the previous model, we denoted the proportion of HP-SHP out of the total intake of the $i$ th horse and the $j$-th day with $q_{i j}$ and we used a logit link function as well. In this model we omitted an intercept term but we considered a flavour coefficient $\alpha_{f}$ based on the citrus flavour to HP proportion, which followed a Normal distribution with mean 0 and variance $\tau_{f}^{2}$. The temporal effects are modeled again as Normal-distributed random variables $\eta_{d j}$ with mean 0 and variance $\tau_{d}^{2}$. Similarly, the random horse effects are Normal-distributed random variables $\eta_{h i}$ with mean 0 and variance $\tau_{h}^{2}$. The residual terms $\xi_{i j}$ followed a Normal distribution with mean 0 and variance $\tau_{\xi}^{2}$ and all the scale hyper-parameters $\tau_{f}^{2}, \tau_{d}^{2}, \tau_{h}^{2}$, followed a Half-Cauchy $(0,1)$ distribution. We also introduced the indicator function $I(i, j)$ to denote if the HP-SHP had a citrus flavour, i.e. $I(i, j)=$ 1 for the $i$-th horse on the $j$-th day or not $(I(i, j)=0)$. The hierarchical model was as follows: 
$\alpha_{f} \sim \operatorname{Normal}\left(0, \tau_{f}^{2}\right) \quad \tau_{f}^{2} \sim$ Half-Cauchy $(0,1) \quad \eta_{d j} \sim \operatorname{Normal}\left(0, \tau_{d}^{2}\right) \quad \tau_{d}^{2} \sim$ Half-Cauchy $(0,1$

$\eta_{h i} \sim \operatorname{Normal}\left(0, \tau_{h}^{2}\right) \quad \tau_{h}^{2} \sim$ Half-Cauchy $(0,1) \quad \xi_{i j} \sim \operatorname{Normal}\left(0, \tau_{\xi}^{2}\right) \quad \tau_{\xi}^{2} \sim$ Half-Cauchy $(0,1)$

$$
\operatorname{logit}\left(q_{i j}\right)=\eta_{h i}+\eta_{d j}+\alpha_{f} I(i, j)+\xi_{i j}
$$

For the implementation of the model a similar approach was used to that described in experiment 1.

\section{Results}

\subsection{Experiment 1: Familiar odour}

The total bamboo consumption $(\mathrm{g}, \mathrm{DM})$ is illustrated with boxplots in Figure 2. The model described in Section 2.7.1 was positively evaluated (see for more details supplementary material) and a greater $\mathrm{BO}^{+}$consumption was found for specific days. Since it is a Bayesian model, a marginal posterior distribution is estimated for each model parameter instead of a point estimate and it is used as the basis of the statistical analysis. The posterior distribution of bamboo consumption (in percentage) at a particular day is summarised in Table 1; we emphasize that these estimates include main, temporal and also random effects. The posterior percentages express the posterior probability as a percentage, i.e. the $\mathrm{BO}^{+}$consumption out of the total intake per day. The contribution of the temporal effects and the main effect to the $\mathrm{BO}+$ consumption is shown in the violin plots (Figure 3), where the random effects are omitted (see supplementary material for more details). On the logistic scale of Figure 3 the posterior mass above zero expresses a higher $\mathrm{BO}^{+}$ consumption. An equal distribution of consumption of $\mathrm{BO}^{-}$and $\mathrm{BO}^{+}$is expressed when the posterior percentage is close to $50 \%$ on Table 1 and a symmetric posterior density centered at zero on Figure 
285 In Table 1 , the first 5 days the $\mathrm{BO}^{+}$accounts for $89.7-100 \%$ of the total consumption whereas at

Day $6 \mathrm{BO}^{-}$accounts for $83.3 \%$ of the total consumption. During Days 7 and 8 we start observing a more balanced distribution between the two choices (the $\mathrm{BO}^{+}$consumption accounted for $65.7 \%$ and $52.9 \%$ of the total intake for Days 7 and 8 respectively) and the $\mathrm{BO}^{+}$consumption increased again on the last day (Day 9, 86.2\%). Additionally, the posterior distribution of the temporal effects for the intake of bamboo (Figure 3) showed a greater consumption for the $\mathrm{BO}^{+}$on Day 2, Day 4 and Day 5 (the corresponding 95\% creditable intervals in Figure 3 are above zero). There is also strong evidence for non-zero temporal effects (Bayes Factor $\mathrm{B}_{21}=110$ in favor of non-zero effects; see details in supplementary material) (Kass and Raftery, 1995).

\subsection{Experiment 2:Nutrient composition (Post-ingestive feedback)}

The total SHP consumption (g, DM) is illustrated with boxplots in Figure 4. The Bayesian model (Section 2.7.2) was assessed in a similar fashion as described in the bamboo experiment, but it received less support from the data (see for more details supplementary material). The distribution of SHP consumption (in percentage) at a particular day is summarised in Table 2, again these estimates include main, temporal and also random effects. Similar to the bamboo data presentation, the posterior probability percentages in Table 2 express the HP-SHP consumption out of the total intake. The contribution of the temporal effects and main effect to the HP-SHP consumptions is shown in the violin plots (Figure 5). When the posterior mass is positioned above zero this indicates a preference towards HP-SHP. When the consumption of HP-SHP and LP-SHP is balanced the posterior percentage is close to $50 \%$ (Table 2) and a symmetric posterior density centered at zero on Figure 5. In order to keep the bulk and fibre content of the two foods similar, we offered $265 \mathrm{~g}$ for the HP-SHP option and $200 \mathrm{~g}$ for the LP-SHP. The added protein meal (65 g) did not contribute to the expansion of the SHP, bonding to the fibrous structure, and we preserved the volume between 
the two options by adding the same amounts of water. We observed total intakes greater than $400 \mathrm{~g}$

311 for Horse 5 on Day 6 and 7 and for Horse 9 on Day 10 and 12 (outliers and whisker Figure 4), and we acknowledge that this a limitation based on weight but not for volume.

With the exception of Horse 5, in Table 2, the random effects indicated a greater consumption of the HP-SHP, which was, on average, between 1.56 to 2.45 times more than the LP-SHP intake. The flavour effect was expressed by $\alpha_{f}$, which was minimal (posterior mean $-0.045 \pm \mathrm{SD}$; 0.216; see details in supplementary material). Initially (in the first week) a variable intake pattern emerged: the first two days indicated a moderate intake for LP-SHP $(\sim 64 \%)$, which was followed by a greater consumption of LP-SHP on Day $3(\sim 85 \%)$ and an equal intake was recorded on Day 5 ( 49\% HP). For the remaining days 4, 6 and 7 a greater consumption of HP-SHP was noted (80-87\%). In the second week, immediately after the flavour switch-over, a higher consumption of LP-SHP was recorded followed by a steady recovery on the remaining days (9-11) to a greater intake of HP-SHP (57 to $81 \%$ ). However, the posterior distribution of the temporal effects for the intake of SHP

(Figure 5) showed large variations and no clear distribution in favor of the HP-SHP was observed (all 95\% creditable intervals cross the zero line in Figure 5).

\section{Discussion}

\subsection{Nutrient composition}

Food acceptance and preference is a result of an interrelationship between the foods flavour (smell, taste and texture) and its post-ingestive consequences (positive or negative) (Provenza, 1995). A previous study in horses suggest that preferences for particular flavours can be overridden by postingestive consequences of a familiar food (Cairns et al., 2002) and this study (second experiment) hypothesised that horses would be similar when they are offered nutritious novel foods; having a 
greater intake of high-protein novel food, regardless of the novel odour (aniseed or citrus). It was also expected that horses would be able to recognise a flavour-protein pairing if the link between the cue and post-ingestive feedback was altered. This study showed a greater posterior percentage for the HP-SHP on days 4, 6 and 7 and after the switch over on days 9 to 14. This suggest that the recognition of the post-ingestive associations (from $\mathrm{CP}$ and/or DE content) initially seemed to take 4 to 5 days which was also apparent in experiment 1 and is in accordance with the findings of Cairns et al. (2002). However, the posterior distribution of the temporal effects (omitting the random effects) for the intake of SHP showed large variations between days and was not supported by the model. This lack of support could be attributed to the dropout in this study, as 4 out of 11 horses did not sample the novel SHP diet within the time frame of the test and were removed from the analysis.

Horses, like ruminants, habitually eat only small quantities of food when it is presented for the first time (Thorhallsdottir et al., 1987; Provenza, 1995; van den Berg et al., 2016). This cautious sampling or even complete rejection of new foods is commonly referred to as neophobia (meaning "the fear of new") and has been suggested as an innate herbivore survival mechanism for avoiding the over-consumption of toxic plants in the wild (Provenza and Balph, 1987). The results from experiment 2 would seem to affirm this pattern. This raises the question of individual variation in the level of neophobia toward novel volatiles (in this case flavours citrus and aniseed) and/or the texture of the SHP. Ott et al. (1979) reported a similar pattern with 6 of 8 mature horses reducing intake of a grain concentrate when it was mixed with $30 \%$ dried citrus pulp consuming only $8.6 \%$ of the feed offered. The strength of neophobia seems to vary considerably between individuals of ruminant species (Launchbaugh et al., 1997; Nolan and Hinch, 1997; Hinch et al., 2004) and this seems to be the case in horses as well. 
363 Food cues (flavour; smell, taste and texture) play an important role in an animal's ability to identify 364 and remember foods with aversive post-ingestive consequences. This has been well documented in 365 ruminants (Provenza et al., 1990; Launchbaugh and Provenza, 1993; Kyriazakis et al., 1997; Pfister 366 et al., 2010) and ponies have been shown to learn to avoid relatively novel foods when apomorphine hydrochloride (APO) was injected intramuscularly to induce illness (Houpt et al., 1990). These authors also showed that ponies could form an aversion to a novel food, based on previous experience, even when it is ingested at the same time as another, more familiar, food. However, this pattern was not completely consistent with high-energy dense foods suggesting that ponies were more likely to develop aversions to less "palatable" foods.

\subsubsection{Odour}

Odour (volatiles) is an important food characteristic, which allows animals to discriminate amongst foods and link these to pleasant or unpleasant experiences. While it is likely that neophobia in experiment 2 was caused by the novel volatiles (aniseed and citrus), we can confirm that a familiar odour can have a positive effect on novel food acceptance. Our study (experiment 1) showed for the first 5 days a posterior percentage between 89.7 and $100 \%$ for the $\mathrm{BO}^{+}$when main, random and temporal effects were included. In addition the posterior distribution of the temporal effects showed that 3 out of 5 days had $95 \%$ creditable intervals that were above zero. There was also strong evidence for temporal effects as the support by the data is 110 times (Bayes factor) greater than the no effects model. This suggests that lucerne odour positively influenced the intake of bamboo during the first 5 days of exposure. After day 5 the intake shifted to an equal $\mathrm{BO}^{+}$and $\mathrm{BO}^{-}$ consumption distribution and horses consumed $50 \%$ or more of the bamboo offered which may suggest that horses had recognised the unfamiliar bamboo as an acceptable food (i.e. lack of a negative post-ingestive feedback). 
It has been shown that animals can generalise preferences and a well-liked familiar flavour can increase the acceptance of a novel food (even if it contain some toxins). Dohi and Yamada (1997) demonstrated that sheep and goats had a greater preference for a less palatable hay when it was sprayed with an extract of a well-liked high-grain concentrate and Van Tien et al. (1999) showed that sheep increased their intake of a novel food (rice bran) more quickly when a familiar grass

suggest a similar pattern for horses. Flavours have been effectively used to encourage intake of water and (medicated) foods by horses. Mars et al. (1992) used apple- or clover-flavours to encourage water acceptance and showed a significant preference for the apple flavour while Burton et al. (1983) reported that apple, lucerne and caramel flavours but not anise-molasses were partially effective in increasing acceptance of a diet containing levamisol or piperazine, and Goodwin et al. (2005) demonstrated that well-liked flavours (fenugreek and banana) significantly reduced relative consumption time of mineral pellets compared to unflavoured pellets. However, these studies have not always clearly defined what type of flavouring (i.e. non-nutritive $v s$. nutritive) was used and if it only affected the smell or also impacted the taste.

In our current study we only used odour as a food cue, which appeared to be strong enough to encourage the acceptance of a nutritious novel food. This suggests that volatiles (pre-ingestive feedback) can play an important role in diet choices by horses and supports the findings of our previous study (van den Berg et al., 2016). The influence of volatiles on the preference of familiar foods (oaten and lucerne) by horses has been previously demonstrated by Pain and Revell (2007; 2009). These authors showed that volatiles can be positively linked to nutritive and physical traits of lucerne or oaten hay. However they also found volatiles that influenced the preference negatively but were not related to any measurable nutritive or physical traits, possibility due to other plant factors such as secondary compounds. This suggests that diet selection by horses cannot always be explained by nutritive traits and that olfaction and gustation cues may also be important. This may 
414 be more apparent when dealing with strong herbaceous volatiles such as from browse species (tree 415 and shrub leaves), which were used in our previous study (van den Berg et al., 2016).

\subsubsection{Taste}

Taste (gustation) could have also influenced the food consumption observed in our study. In

experiment 2 we added soybean meal, which may have contributed to a greater acceptance of the HP-SHP option. It has been shown that horses can distinguish between 4 of the 5 taste sensations (sweet, bitter, salty and sour) (Randall et al., 1978), but there may even be an indication that horses could also have a taste for "umami". Umami is a Japanese loanword and can be translated as "pleasant savoury taste". Umami perception occurs through the detection of the carboxylate anion of glutamic acid, which is a naturally occurring amino acid common in meats, cheese, broth, stock and other protein-heavy foods (Chandrashekar et al., 2006). While umami taste responses has been mainly linked to a carnivorous or omnivorous diet, it seems that herbivores such as horses and cattle express also the taste receptor genes involved with the umami taste sensation (Zhao et al., 2010). In addition, Favreau et al. (2010) has demonstrated in sheep that an umami taste resulted in a greater intake/preference compared to a bitter taste and Bach et al. (2012) showed that sheep form preferences for umami-flavoured feeds, even when those feeds are novel and low in CP. This could indicate that horses may also have the ability to detect foods with a "protein taste" which could explain why horses seem to have a greater preference for HP-SHP diet. However, to our knowledge no studies are known that have explicitly assessed this in horses, therefore in hindsight it could have been noteworthy to examine how taste (sweet or umami) could have influenced the neophobia seen in this study.

\subsubsection{Texture}

The texture and ease of prehension are also food characteristics that can influence the food 
the soybean processing industry which contain 53-70\% neutral detergent fibre (NDF) and less than $4413 \%$ starch (NRC, 2007). It is considered to be a good alternative fibre-product for inclusion in 442 livestock feeds and has a growing use in commercial horse feeds due to its low glycaemic index 443 (GI) profile. While it is high in digestible fibre its initial palatability may be modest when it is fed on its own which could have attributed to the texture or taste. When fed dry SHP can be dusty and when dampened the fibres expand which result in a "cottony" texture. Overall texture and/or palatability could have influenced the horse's foraging behaviour in our study.

\section{Conclusion}

The findings of the present study confirm that horses use organoleptic qualities of forages (e.g. odour, taste, texture, ease of prehension etc.) when selecting foods. Horses in this study were able to make an association with the nutritional content of a novel food, but neophobia significantly impacted on initial testing of novel foods. We were also able to show that the acceptance of a novel food can be enhanced by introducing a familiar odour, which highlights the important role of the pre-ingestive signals in diet selection by horses. The present study was not able to examine the role of taste in dietary preferences by horses, as non-nutritive flavours (odours) were used. Future preference studies should incorporate odour, taste and texture to determine the relative importance of these "cues" to horses making diet choices in grazing environments. Nevertheless, the present study adds to the understanding of mechanisms involved in diet selection by horses and proposes that odour cues can be successfully applied in equine feeding management when forages/foods are scarce and new foods or forage batches are introduced or when horses are moved to different environments. 
The care and use of the animals followed the guidelines set by The University of New England

\section{Conflict of interest statement}

None.

\section{Acknowledgements}

Funding for this project was kindly provided by the University of New England. The authors thank the Todd Family for their kind assistance in providing horses and facilities. The authors also acknowledge Karina McLoughlan, Kelly McLean, Brittany McVicar and Amber Alsford for assisting during the experimental work, and Michael Raue for technical support. Special thanks go to our sponsors; BEC Feed Solutions and Energreen Nutrition Australia.

\section{References}

Arnold, G.W., Boer, E.S., Boundy, C.A.P., 1980. The influence of odour and taste on the food preferences and food intake of sheep. Aust. J. Agric. Res. 31, 571-587.

Bach, A., Villalba, J., Ipharraguerre, I., 2012. Interactions between mild nutrient imbalance and taste preferences in young ruminants. J. Anim. Sci. 90, 1015-1025.

Burritt, E.A., Provenza, F.D., 1991. Ability of lambs to learn with a delay between food ingestion and consequences given meals containing novel and familiar foods. Appl. Anim. Behav. Sci. 32, 179-189.

Burton, J.H., Price, D.J., Aspinal, J., 1983. The effect of feed flavour and feed consumption in horses, In: Proceedings of the 8th Equine Nutrition and Physiology Symposium, Lexington, Kentucy, USA, pp. 27-32.

Cairns, M.C., Cooper, J.J., Davidson, H.P.B., Mills, D.S., 2002. Association in horses of orosensory characteristics of foods with their post-ingestive consequences. Anim. Sci. 75, 257-265.

Chandrashekar, J., Hoon, M.A., Ryba, N.J.P., Zuker, C.S., 2006. The receptors and cells for mammalian taste. Nature 444, 288-294.

Cuddeford, D., 2005. Voluntary food intake by equids. Pferdeheilkunde 21, 7-8.

Dohi, H., Yamada, A., 1997. Preference of sheep and goats for extracts from high-grain concentrate. J. Anim. Sci. 75, 2073-2077. 
Dulphy, J.P., Martin-Rosset, W., Dubroeucq, H., Ballet, J.M., Detour, A., Jailler, M., 1997. Compared feeding patterns in ad libitum intake of dry forages by horses and sheep. Livest. Prod. Sci. 52, 49-56.

499 Favreau, A., Baumont, R., Ferreira, G., Dumont, B., Ginane, C., 2010. Do sheep use umami and 500 bitter tastes as cues of post-ingestive consequences when selecting their diet? Appl. Anim. Behav. 501 Sci. 125, 115-123.

502 Garcia, J., McKowan, B.K., Green, K.F., 1972. Biological constraints on conditioning, in: 503 Seligman, M.E.P., Hager, J.L. (Eds.), Biological boundaries of learning, Meredith Corporation 504 Company (Appleton-Century-Crofts, Inc.), New York, USA, pp. 21-43.

Gelman, A., 2006. Prior distributions for variance parameters in hierarchical models (comment on 506

Goodwin, D., Davidson, H.P.B., Harris, P., 2005. Selection and acceptance of flavours in concentrate diets for stabled horses. Appl. Anim. Behav. Sci. 95, 223-232.

Henneke, D., Potter, G.D., Kreider, J.L., Yeated, B.F., 1983. Relationship between condition score, physical measurments and body fat percentage in mares. Equine Vet. J. 15, 371-372.

Hinch, G.N., Nolan, J.V., Lynch, J.J., Hill, J., 2004. Familiar odour and flavour cues reduce feed neophobia in sheep. Anim. Prod. Aus. 25, 97-99.

Houpt, K.A., Zahorik, D.M., Swartzman-Andert, J.A., 1990. Taste aversion learning in horses. J. Anim. Sci. 68, 2340-2344.

Kass, R.E., Raftery, A.E., 1995. Bayes factors. J. Amer. Statist. Assoc. 90, 773-795.

Kyriazakis, I., Papachristou, T.G., Duncan, A.J., Gordon, I.J., 1997. Mild conditioned food aversions developed by sheep towards flavors associated with plant secondary compounds. J. Chem. Ecol. 23, 727-746.

Launchbaugh, K.L., Provenza, F.D., 1993. Can plants practice mimicry to avoid grazing by mammalian herbivores? Oikos 66, 501-504.

Launchbaugh, K.L., Provenza, F.D., Werkmeister, M.J., 1997. Overcoming food neophobia in Appl. Anim. Behav. Sci. 54, 327-334.

Laut, J.E., Houpt, K.A., Hintz, H.F., Houpt, T.R., 1985. The effects of caloric dilution on meal patterns and food intake of ponies. Physiol. Behav. 35, 549-554.

Mars, L.A., Kiesling, H.E., Ross, T.T., Armstrong, J.B., Murray, L., 1992. Water acceptance and intake in horses under shipping stress. J. Equine Vet. Sci. 12, 17-20.

Nelson, G., Keller M, Cheeke, P.R., 1997. Evaluation of temperate bamboo species as forage for livestock, Pacific Northwest Bamboo Agroforestry Workshop Port Townsend, Washington, USA, pp. 7-1 to 7-8.

Nolan, J.V., Hinch, G.N., 1997. Factors that determine whether grazing animals will accept supplements. In: Proceedings of Recent Advances in Animal Nutrition in Australia, Armidale, NSW, Australia, pp. 55-62. 
NRC, 2007. Nutrient requirements of horses. 6th edition ed. The National Academies Press, Washington D.C., USA.

Ott, E.A., Feaster, J.P., Lieb, S., 1979. Acceptability and digestibility of dried citrus pulp by horses. J. Anim. Sci. 49, 983-987.

Pain, S., Revell, D.K., 2007. Natural odorants can influence the preference of horses for lucerne hay. In: Proceedings of Recent Advances in Animal Nutrition in Australia, Armidale, NSW, Australia, pp. 27-33.

Pain, S., Revell, D.K., 2009. Fodder quality specifications: Identifying predictors of preference between hays. Rural Industries Research and Development Corporation, Kingston, ACT, Australia.

Pfister, J.A., Gardner, D.R., Cheney, C.C., Panter, K.E., Hall, J.O., 2010. The capability of several toxic plants to condition taste aversions in sheep. Small Ruminant Res. 90, 114-119.

Pfister, J.A., Stegelmeier, B.L., Cheney, C.D., Ralphs, M.H., Gardner, D.R., 2002. Conditioning taste aversions to locoweed (Oxytropis sericea) in horses. J. Anim. Sci. 80, 79-83.

Plummer, M., 2003. JAGS: A program for analysis of Bayesian graphical models using Gibbs sampling, Proceedings of the 3rd International Workshop on Distributed Statistical Computing, Vienna, Austria.

Plummer, M., Stukalov, A., 2014. rJAGS-package: Bayesian graphical models using MCMC, Interface to the JAGS MCMC library. http://mcmc-jags.sourceforge.net/

Provenza, F.D., 1995. Postingestive feedback as an elementary determinant of food preference and intake in ruminants. J. Range Manage. 48, 2-17.

Provenza, F.D., 1996. Acquired aversions as the basis for varied diets of ruminants foraging on rangelands. J. Anim. Sci. 74, 2010-2020.

Provenza, F.D., Balph, D.F., 1987. Diet learning by domestic ruminants: Theory, evidence and practical implications. Appl. Anim. Behav. Sci. 18, 211-232.

Provenza, F.D., Burritt, E.A., Clausen, T.P., Bryant, J.P., Reichardt, P.B., Distel, R.A., 1990. Conditioned Flavor Aversion: A Mechanism for Goats to Avoid Condensed Tannins in Blackbrush. Am. Nat. 136, 810-828.

Randall, R.P., Schurg, W.A., Church, D.C., 1978. Response of horses to sweet, salty, sour and bitter solutions. J. Anim. Sci. 47, 51-55.

Redgate, S.E., Cooper, J.J., Hall, S., Eady, P., Harris, P.A., 2014. Dietary experience modifies horses' feeding behavior and selection patterns of three macronutrient rich diets. J. Anim. Sci. 92, 1524-1530.

Spiegelhalter, D.J., Best, N.G., Carlin, B.P., Van Der Linde, A., 2002. Bayesian measures of model complexity and fit. J. R. Stat. Soc. Series B Stat. Methodol. 64, 583-639.

Team, R.D.C., 2015. R: A language and environment for statistical computing, R Foundation for Statistical Computing, Vienna, Austria. https://www.r-project.org/

Thorhallsdottir, A.G., Provenza, F.D., Balph, D.F., 1987. Food aversion learning in lambs with or without a mother: Discrimination, novelty and persistence. Appl. Anim. Behav. Sci. 18, 327-340. 
572 Triebe, C., Van den Berg, M., Brown, W.Y., 2012. Tagasaste versus Golden Bamboo: Which do 573 horses prefer? In: Proceedings of the Australasian Equine Science Symposium, Gold Coast, 574 Australia, p. 41.

575 van den Berg, M., Lee, C., Brown, W.Y., Hinch, G.N., 2016. Does energy intake influence diet 576 selection of novel forages by horses? Livest. Sc. 186, 6-15.

577 Van Tien, D., Lynch, J.J., Hinch, G.N., Nolan, J.V., 1999. Grass odor and flavor overcome feed 578 neophobia in sheep. Small Ruminant Res. 32, 223-229.

579 Zhao, H., Yang, J.-R., Xu, H., Zhang, J., 2010. Pseudogenization of the umami taste receptor gene 580 Tas1r1 in the giant panda coincided with its dietary switch to bamboo. Mol. Biol. Evol. 27, 26695812673. 
Table 1: A Bayesian hierarchical model was used to determine the effect of a familiar odour (lucerne) on the intake of novel forages (bamboo). In this table the estimated posterior percentage of bamboo with lucerne odour consumption out of the total intake are presented. These estimates include main, temporal (i.e. day) and random (i.e. horse) effects.

Table 2: A Bayesian hierarchical model was used to establish if horses make associations with the orosensory characteristics of a novel food (soybean hull pellets) and their postingestive consequences (protein levels). In this table the estimated posterior percentage of high-protein soybean hull pellets consumption out of the total intake are presented. These estimates include main, temporal (i.e. day) and random (i.e. horse) effects.

Figure 1. Timeline (day) of experiments.

Figure 2. Experiment 1: Boxplot of the total bamboo intake (g, DM) over the 9 testing days $(\mathbf{n}=11)$.

Figure 3. A Bayesian hierarchical model was used to determine the effect of a familiar odour (lucerne) on the intake of novel forages (bamboo). In this figure the violin plots of the posterior distribution of $95 \%$ credible intervals in logistic scale of the temporal effects (i.e. day) for the intake of bamboo with lucerne odour $\left(\mathrm{BO}^{+}\right)$are presented $(\mathrm{n}=11)$. When the posterior mass is positioned above zero this expresses a higher $\mathrm{BO}^{+}$ consumption. 
Figure 4. Experiment 2: Boxplot of the total soybean hull pellets intake over 14 testing days (flavour-diet combination was switch at the end of week 1$)(n=7)$.

Figure 5. A Bayesian hierarchical model was used to establish if horses make associations with the orosensory characteristics of a novel food (soybean hull pellets) and their post-ingestive consequences (protein levels). In this figure the violin plots of the posterior distribution of $95 \%$ credible intervals in logistic scale of the temporal effects (i.e. day) for the intake of high-protein soybean hull pellets (HP-SHP) are presented $(n=7)$. When the posterior mass is positioned above zero this indicates a preference towards HP-SHP. 
Table 1: A Bayesian hierarchical model was used to determine the effect of a familiar odour (lucerne) on the intake of novel forages (bamboo). In this table the estimated posterior percentage of bamboo with lucerne odour consumption out of the total intake are presented. These estimates include main, temporal (i.e. day) and random (i.e. horse) effects.

\begin{tabular}{ccccccccc}
\multicolumn{10}{c}{ Days } \\
\hline 1 & 2 & 3 & 4 & 5 & 6 & 7 & 8 & 9 \\
\hline $94.9 \%$ & $97.7 \%$ & $89.7 \%$ & $100.0 \%$ & $99.9 \%$ & $16.7 \%$ & $65.7 \%$ & $52.9 \%$ & $86.2 \%$ \\
\hline
\end{tabular}


Table 2: A Bayesian hierarchical model was used to establish if horses make associations with the orosensory characteristics of a novel food (soybean hull pellets) and their post-ingestive consequences (protein levels). In this table the estimated posterior percentage of highprotein soybean hull pellets consumption out of the total intake are presented. These estimates include main, temporal (i.e. day) and random (i.e. horse) effects. Flavour-protein pairing was switched over after week 1.

\begin{tabular}{cccccccc}
\hline & $\mathbf{1}$ & $\mathbf{2}$ & $\mathbf{3}$ & $\mathbf{4}$ & $\mathbf{5}$ & $\mathbf{6}$ & $\mathbf{7}$ \\
\hline Week 1 & $36.1 \%$ & $36.4 \%$ & $14.8 \%$ & $79.6 \%$ & $48.6 \%$ & $82.5 \%$ & $87.0 \%$ \\
& & & & & & & \\
\hline Week 2 & $\mathbf{8}$ & $\mathbf{9}$ & $\mathbf{1 0}$ & $\mathbf{1 1}$ & $\mathbf{1 2}$ & $\mathbf{1 3}$ & $\mathbf{1 4}$ \\
\hline
\end{tabular}




\section{Experiment 2:}

Two-choice test presenting a novel food high and low in protein paired with one of two unfamiliar (non-nutritive) odours

\section{Familiar forage Experiment 1:}

(testing area Two-choice test presenting a novel

adaptation) forage with and without a familiar odour
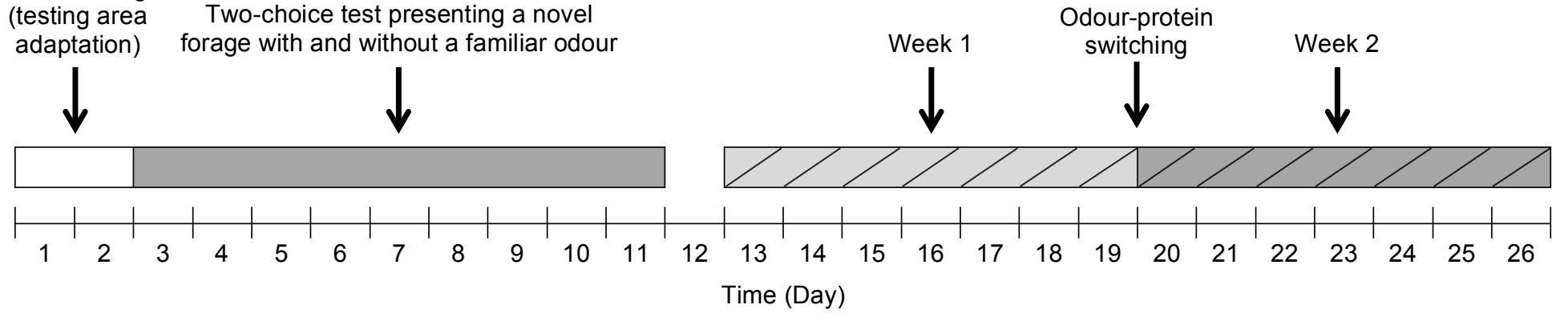
Figure 3: Violin plots of posterior distribution (Experiment 1)

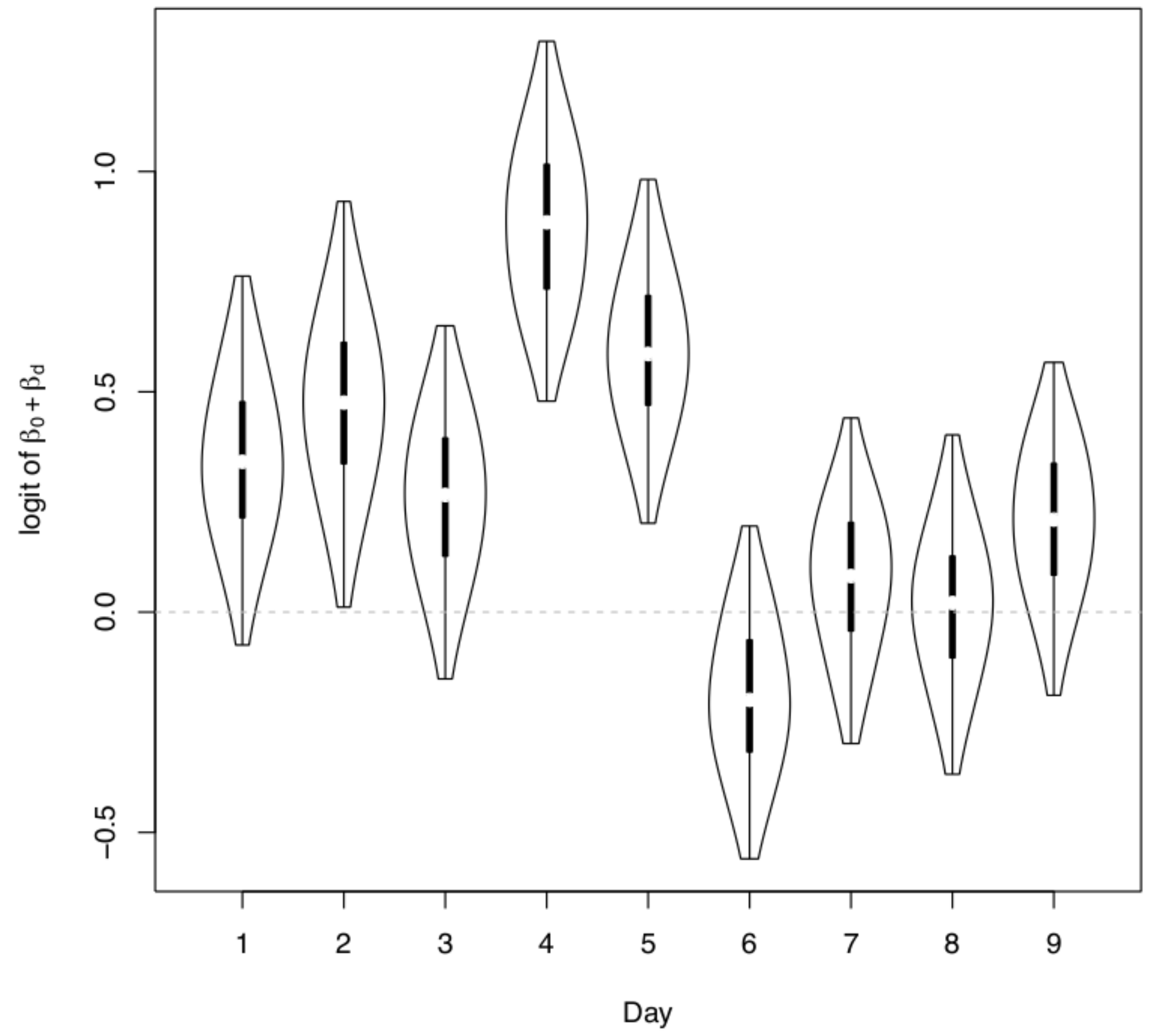


Figure 5: Violin plots of posterior distribution (Experiment 2)

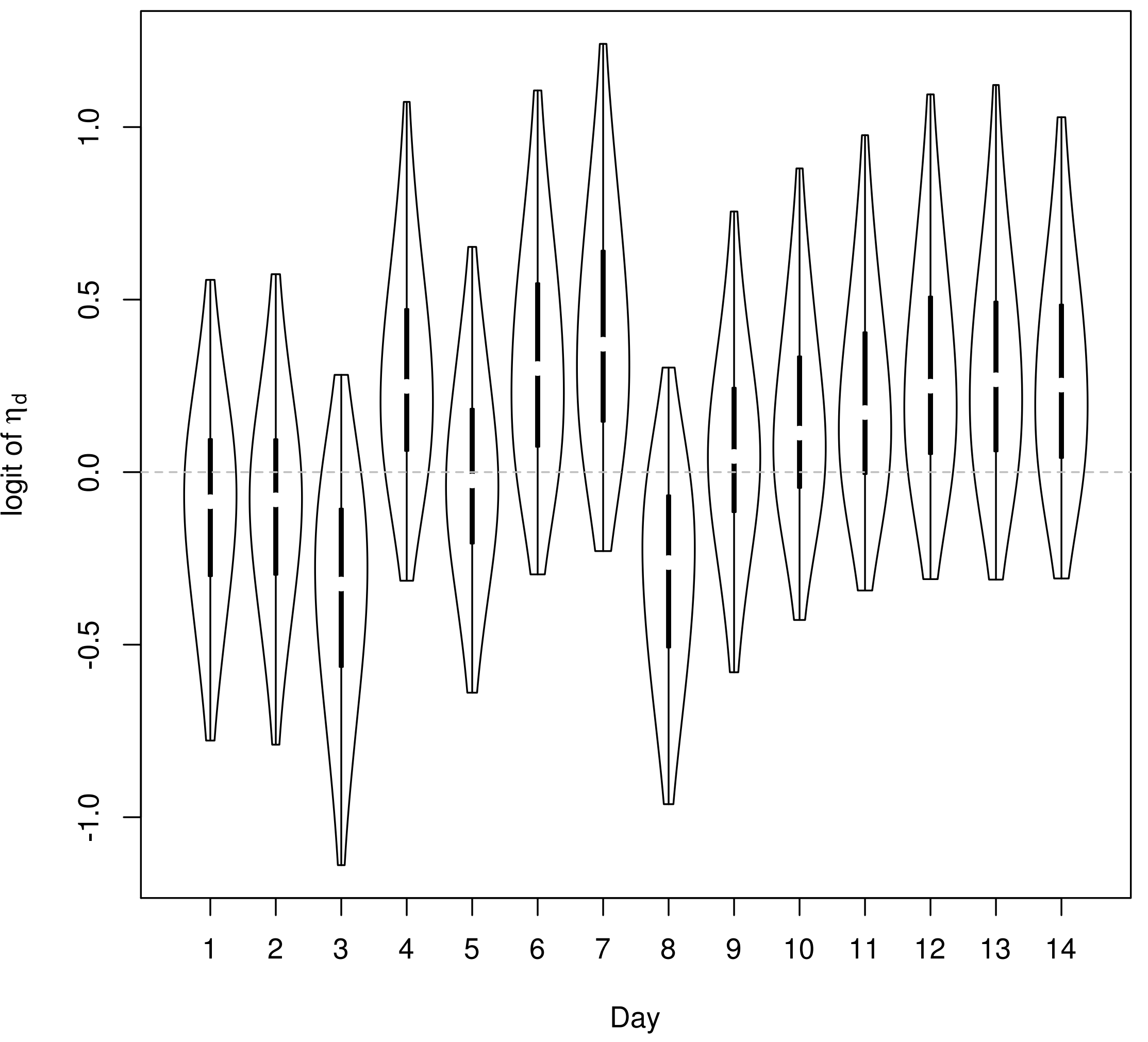

